\title{
Non-destructive Measurement of Photoexcited Carrier Transport in Graphene with Ultrafast Grating Imaging Technique
}

Ke Chen, ${ }^{\text {a,b }}$ Maruthi Nagavalli Yogeesh, ${ }^{\mathrm{c}}$ Yuan Huang, ${ }^{\mathrm{d}}$ Shaoqing Zhang, ${ }^{\mathrm{b}}$ Feng He, ${ }^{\mathrm{b}, \mathrm{e}}$ Xianghai Meng, ${ }^{\mathrm{b}}$ Shaoyin Fang, ${ }^{\mathrm{a}}$ Nathanial Sheehan, ${ }^{\mathrm{c}}$ Tiger Hu Tao, ${ }^{\mathrm{b}}$ Seth R. Bank, ${ }^{\mathrm{c}}$ Jung-Fu Lin, ${ }^{\text {f,g }}$ Deji Akinwande, ${ }^{\mathrm{c}}$ Peter Sutter, ${ }^{\mathrm{h}}$ Tianshu Lai, ${ }^{\mathrm{a}, *}$ Yaguo Wang ${ }^{\mathrm{b}, \mathrm{e},}$

a. State-Key Laboratory of Optoelectronic Materials and Technologies, School of Physics and Engineering, Sun Yat-Sen University, Guangzhou, Guangdong 510275, China

b. Department of Mechanical Engineering, The University of Texas at Austin, Austin, TX, 78712, USA

c. Department of Electrical and Computer Engineering, Microelectronics Research Center, The University of Texas at Austin, Austin, TX, 78758, USA

d. Center for Functional Nanomaterials, Brookhaven National Lab, Upton, NY, 11973, USA

e. Texas Materials Institute, The University of Texas at Austin, Austin, TX, 78712, USA

f. Department of Geological Sciences, Jackson School of Geosciences, The University of Texas at Austin, Austin, TX, 78712, USA

g. Center for High Pressure Science and Technology Advanced Research (HPSTAR), Shanghai 201900, China

h. Department of Electrical \& Computer Engineering, University of Nebraska-Lincoln, Lincoln, NE, 68588, USA

\footnotetext{
* Corresponding authors.

E-mail address: yaguowang@ austin.utexas.edu (Yaguo Wang)

E-mail address: stslts@mail.sysu.edu.cn (Tianshu Lai)
} 


\section{ABSTRACT:}

Graphene has great potential for fabrication of ultrafast opto-electronics, in which relaxation and transport of photoexcited carriers determine device performance. Even though ultrafast carrier relaxation in graphene has been studied vigorously, transport properties of photoexcited carriers in graphene are largely unknown. In this work, we utilize an ultrafast grating imaging technique to measure lifetime $\left(\boldsymbol{\tau}_{\boldsymbol{r}}\right)$, diffusion coefficient $(\boldsymbol{D})$, diffusion length $(\boldsymbol{L})$ and mobility $(\boldsymbol{\mu})$ of photoexcited carriers in monoand multi-layer graphene non-invasively. In monolayer graphene, $\boldsymbol{D} \sim 10,000 \mathrm{~cm}^{2} / \mathrm{s}$ and $\boldsymbol{\mu} \sim 120,000 \mathrm{~cm}^{2} / \mathrm{Vs}$ have been observed, both of which decrease drastically in multilayer graphene, indicating that the remarkable transport properties in monolayer graphene originate from its unique Dirac-Cone energy structure. Mobilities of photoexcited carriers measured here are several times larger than the Hall and Field-Effect mobilities reported in literature $\left(<15,000 \mathrm{~cm}^{2} / \mathrm{Vs}\right)$, due to the high energy of photoexcited carriers. Our results indicate the importance of obtaining monolayer graphene to realize high-performance graphene devices, as well as the necessity to use transport properties of photoexcited carriers for predicting the performance of graphene-based opto-electronics. 


\section{Introduction}

Graphene has great potential application in high-speed electronics due to its unique electronic properties. Carrier transport properties, such as mobility $\mu$, and diffusion length $L$ determine performance of graphene devices. Electrons in graphene have been found to possess ultrahigh carrier mobility under an external electric field [1-4]. Historically, carrier mobility used for electronics is usually measured via the Hall Effect, where a magnetic field is used to detour electrons [5]. For graphene-based Field-Effect-transistors, Field-Effect mobility is used, where the source-drain current is measured under a gate voltage, either at linear or saturation regions [6].Both aforementioned methods are environmental-temperature static measurements and deposition of electrodes is required.

While carrier mobility $\mu$ measures how fast carriers can travel under an electrical driving force, carrier diffusion length $L$ determines how far carriers can travel without the driving force. $L$ can be calculated with known carrier lifetime $\tau_{r}$ and diffusion coefficient $D: L=\sqrt{D \tau_{r}}$. Due to random scattering motion, carrier diffusion is usually much slower than the carrier drift process driven by an electric field. Consequently, carrier diffusion actually plays a more important role in determining some key properties of traditional p-n junction based opto-electric devices, such as the response rate of photodiodes, and the efficiency of LEDs and photovoltaics. Recently, lateral graphene/hBN heterostructrues have been synthesized, an important step towards developing atomically thin planar integrated circuitry $[7,8]$. Two dimensional transistor with the lateral graphene/hBCN/graphene structure has been demonstrated to exhibit 
an $I_{\mathrm{on}} / I_{\text {off }}$ ratio larger than $10^{4}$, overcoming the main drawback of the current graphene transistor [9]. Another type of combination of graphene with 2D materials is the van der Waals (vdW) heterostructures, where graphene is bonded to a thin film of transition metal dichalcogenides (TMD) by the vdW force and shows ultrafast electron transfer and strong interlayer coupling between graphene and TMD [10]. On the platforms of the aforementioned heterostructures, carrier diffusion in graphene becomes very important. Due to the huge difference in resistance between the hetero-junction and the graphene, almost all the applied voltage is exerted on the junctions or the stack area such that carrier transportation in the graphene region outside the junction or far away from the stack area mainly relies on the diffusion process. Therefore, the carrier diffusion property in graphene turns out to be a key parameter that determines the performance of future electronic devices based on graphene/2D material heterostructures.

Carrier diffusion lengths of semiconductors are usually measured via Electron-beam-induced current (EBIC) [11], light-induced current (LIC) [12], or from spectral response data [13]. EBIC and LIC also require deposition of electrodes, which is intrinsically invasive, and their sensitivity and accuracy are highly affected by electrical contact resistance and the capability to detect small current signals. Determination of $L$ from spectral response data requires accurate values of spectral absorption length, which is not trivial to obtain. For graphene applications in optoelectric devices [14], such as ultrafast switches, saturable absorbers, ultrafast photodetectors, or even solar cells, mobility and diffusion length of photoexcited car- 
riers, rather than intrinsic carriers, should be used. Due to the ultrafast nature of photoexcited carriers in graphene, traditional measurements are not capable to measure transport properties of the excited carriers that only live for ps to ns time scales.

Ultrafast laser pump-probe spectroscopy has emerged as a powerful tool to investigate the carrier relaxation dynamics in graphene [15-22]. The reported ultrafast optical response of graphene, i.e. the differential transmission signal $\Delta \mathrm{T} / \mathrm{T}_{0}$, shows a great diversity in literature: fully positive [16], fully negative $[18,22]$, and first positive and then negative $[20,21] \Delta \mathrm{T} / \mathrm{T}_{0}$ signals have been reported. The diversity can be due to the possible differences in Fermi energy, scattering time and the probed level for different samples [23]. However, measurement of ultrafast dynamics of photoexcited carrier transport in graphene has been rare, only reported by Ruzicka et al. with spatial scanning microspectroscopy $[24,25]$. A possible reason for the very few investigations on this topic is the insufficiency of simple and sensitive measuring methods. Besides the spatial scanning microspectroscopy [24], two other types of optical methods have been applied to measure diffusion coefficient of photoexcited carrier: transient grating diffraction [26] and mask-modulated spectroscopy [27, 28]. However, these two methods suffer the disadvantages of weak signal intensity and invasive contact to sample, respectively, which hinders the experimental studies on photoexcited carrier transport in graphene as well as other 2D materials.

In this article, we present a simple, non-invasive and highly sensitive technique to measure ultrafast photoexcited carrier transport in graphene. We have developed a 
model for the method to analyze the ultrafast spectroscopic signals and to determine the carrier lifetime $\left(\boldsymbol{\tau}_{\boldsymbol{r}}\right)$, diffusion coefficient $(\boldsymbol{D})$, diffusion length $(\boldsymbol{L})$ and mobility $(\boldsymbol{\mu})$ in CVD-grown and exfoliated mono- and multi-layer graphene non-invasively. In monolayer graphene, $\boldsymbol{D} \sim 10,000 \mathrm{~cm}^{2} / \mathrm{s}$ and $\boldsymbol{\mu} \sim 120,000 \mathrm{~cm}^{2} / \mathrm{Vs}$ have been observed, both of which decrease drastically in multilayer graphene, indicating that the remarkable transport properties in monolayer graphene originate from its unique Dirac-Cone energy structure. Mobilities of photoexcited carriers measured here are several larger than the Hall and Field-Effect mobilities reported in literature $\left(<15,000 \mathrm{~cm}^{2} / \mathrm{Vs}\right)$, due to the high energy of photoexcited carriers. Our results indicate the importance of obtaining monolayer graphene to realize high-performance graphene devices, as well as the necessity to use transport properties of photoexcited carriers for predicting the performance of graphene-based opto-electronics.

\section{Experimental section}

Our new technique is inspired by the grating-mask technique [27] but avoids the shortcomings from sample-grating contact. As shown in Fig. 1, pump and probe beams overlap at a same spot on the grating (photomask), whose reduced scale image is formed by an objective lens onto the sample plane, so that the intensities of pump and probe spots at the sample are modulated to be in the grating-pattern. As a result, an initial carrier density with grating-like spatial profile is generated by the pump pulse. And the probe pulse can only detect the excited carriers in the illuminated area, 
which will decay due to both electron-hole recombination and ambipolar carrier diffusion from the illuminated area to the unilluminated area. Therefore, by recording the time-resolved transmission change of probe $(\Delta \mathrm{T})$, information of both carrier recombination $\left(\tau_{r}\right)$ and carrier diffusion $(D)$ processes is obtained. Carrier recombination lifetime $\tau_{r}$ can be easily acquired from the conventional pump-probe experiment [15, 17] by simply removing the grating. A model based on diffusion equation and the relation between differential transmission $\Delta \mathrm{T}$ and carrier density needs to be derived to extract diffusion coefficient $D$.

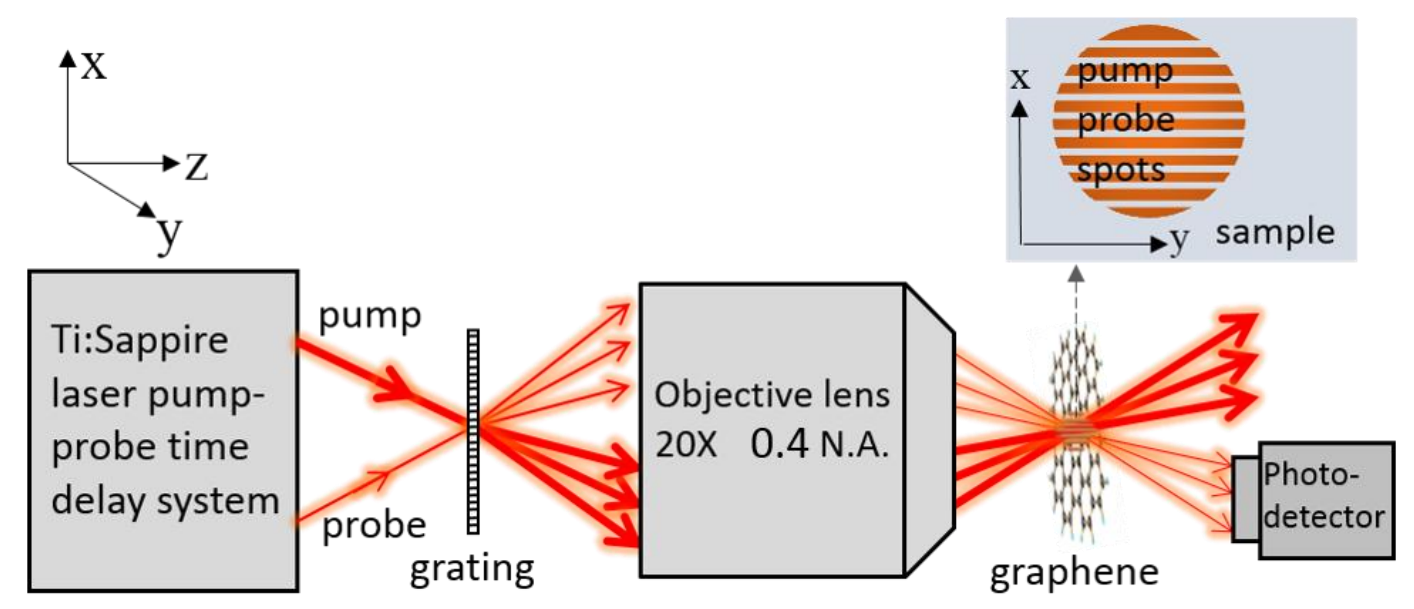

Fig. 1. Schematics of the ultrafast grating imaging technique for probing the diffusion coefficient of graphene. $800 \mathrm{~nm}$ laser beam generated from Ti/Sapphire oscillator, is split into a pump beam and a probe beam, both of which are spatially focused and overlapped into a $90 \mu \mathrm{m}$ spot (diameter) on the grating. Grating images from both pump and probe beams are formed at the sample plane by an objective lens. The intensities of pump and probe beams on the sample are thus modulated as the grating pattern. The transmitted probe beams are collected by a photodetector.

There are several advantages in this method. First, the strong transmission change of probe beam, instead of the weak diffraction, is collected as signal. Second, only two measurements (with and without grating) need to be performed to retrieve 
transport parameters. Third, unlike the electrical measuring techniques which requires electrode deposition or optical mask-modulation approach, the sample here is free of any undesired contact. The above features make the technique sensitive, simple, and truly non-destructive. A key parameter for this technique to work effectively is the ratio $R$ of diffusion length $L$ to image slit width $W_{s}$, i.e. $R=L / W_{s}$. The smallest image slit width $W_{\text {smin }}$ that can be obtained in our setup is estimated from the resolution of microscope objective lens: $0.61 \lambda / N . A$. The numerical aperture N.A. is 0.4 , and the typical working wavelength of Ti:Sapphire laser oscillator is $800 \mathrm{~nm}$. Thus in principle, $W_{s \min }$ in the system is $1.2 \mu \mathrm{m}$. If $R<0.1$ is set to be the failure criterion, then the technique should be able to detect the diffusion effect originating from a diffusion length short to $120 \mathrm{~nm}$ (see supplementary information for determination of the sensitivity of the technique).

Four graphene samples are studied here. One of them is obtained by mechanical exfoliation with Scotch tape [29], two of them are CVD grown on copper foil, and then transferred to quartz substrate (see supplementary for details), the fourth one is bilayer obtained by stacking two CVD grown monolayers. By using atomic force microscope (AFM) and Raman spectroscopy, we have determined that the exfoliated sample has 18 layers with $\sim 6 \mathrm{~nm}$ thickness (Fig. S2), the multilayer CVD sample has 12 layers with $\sim 4 \mathrm{~nm}$ thickness (Fig. S1), and another CVD sample is monolayer with single Lorentzian shape of 2D peak and large $2 \mathrm{D} / \mathrm{G}$ peak ratio in the Raman spectrum (Fig. S4). The bilayer character of the stacked sample is confirmed by Raman spectrum, with $2 \mathrm{D} / \mathrm{G}$ peak ratio close to 1 and a wider $2 \mathrm{D}$ peak than the monolayer (Fig. 
S5). The AFM and Raman mapping data also show good uniformity of our samples

(Fig. S1-S3). Fig. 2(a-c) shows ultrafast transmission signals of 12-layer, 18-layer and bilayer samples, with two grating images of different slit widths. For monolayer graphene, only one grating slit has been used, with measurements done at various pump fluences, as shown in Fig. 2(d). Both pump and probe pulses have 800nm center wavelengths, $500 \mathrm{fs}$ pulse widths and $80 \mathrm{MHz}$ repetition rates. The diameters of pump and probe spots on sample surface are $90 \mu \mathrm{m}$. All graphene samples display a positive peak followed by a negative recovery part. While the positive peak originates from a decrease in the interband conductivity due to state filling of un-thermalized electrons, the negative signal reflects the increase in the intra-band conductivity due to the free carrier absorption $[20,21,30]$. The values of positive peaks are the same with and without grating, showing that the effective excited carrier densities in the illuminated area are the same. However, compared to the no-grating case, the magnitudes of negative peaks with gratings are smaller, and the narrower the grating slit is, the smaller the peak. This phenomenon reveals the carrier diffusion effect in graphene. Due to the rule of intra-band transition, the magnitude of the negative signal is qualitatively correlated with the free carrier density $N$ and the electron temperature $T_{e}$. With gratings, carrier diffusion from the illuminated area to the dark area leads to faster decays of $N$ and $T_{e}$ in the illuminated (probed) area than that in the no grating case, and consequently, magnitudes of negative peaks become smaller compared to the no grating case. In addition, with narrower grating slit, contribution to carrier decay from the diffusion process increases, which results in further decease of 
the negative signal. Note that in Fig. 2(c), differential transmission signal of the quartz

substrate is also plotted. Compared with graphene, the substrate has negligible signal,

which confirms that the transient data purely reflects carrier dynamics in graphene.
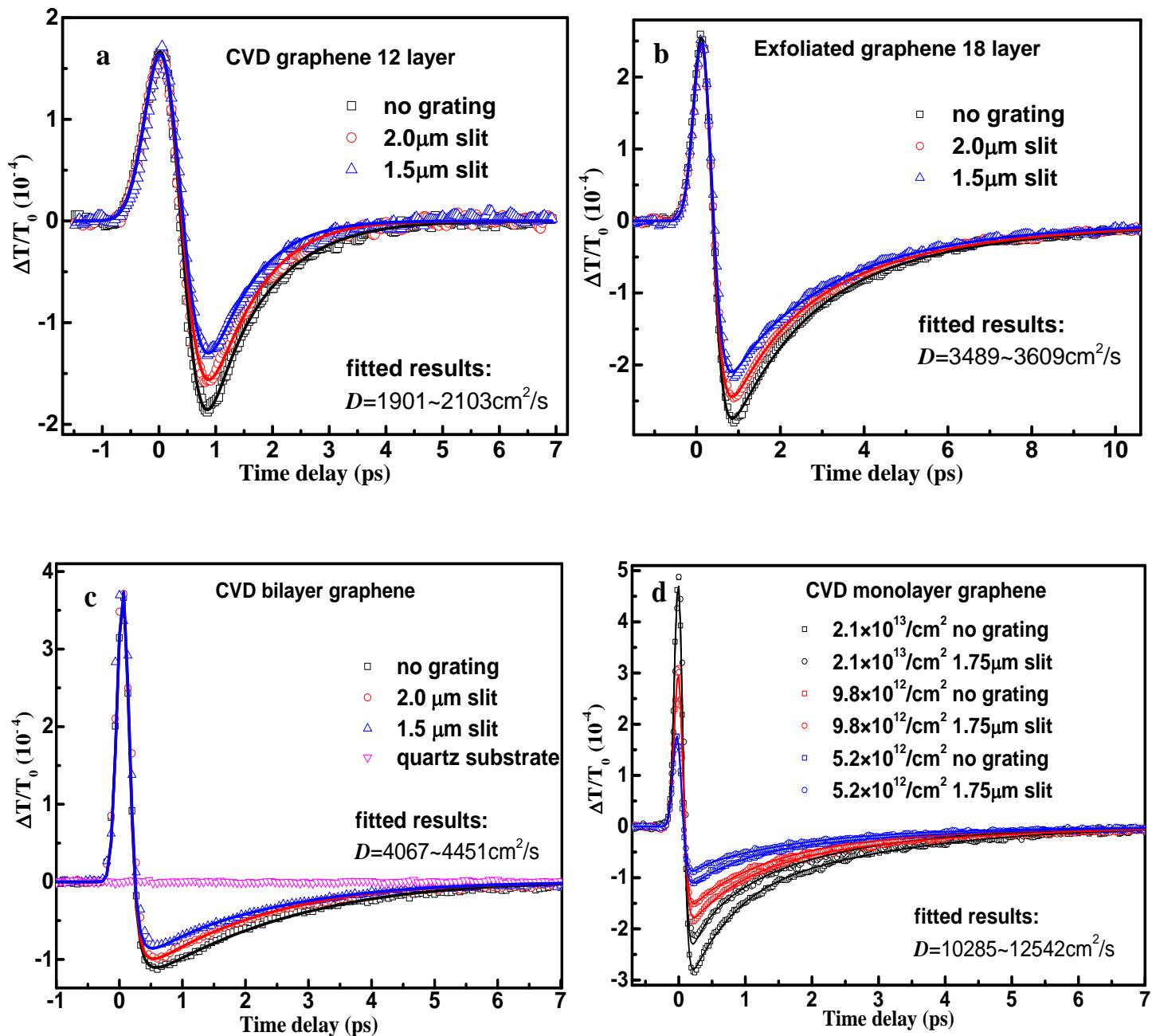

Fig.2. (a) Differential transmission signal of CVD grown 12layer graphene sample measured with ultrafast grating imaging technique at excited carrier density of $2.3 \times 10^{13} / \mathrm{cm}^{2}$. (b) Differential transmission signal of exfoliated 18layer graphene sample measured at excited carrier density of $2.2 \times 10^{13} / \mathrm{cm}^{2}$. (c) Differential transmission signal of CVD grown bilayer graphene sample measured with ultrafast grating imaging technique at excited carrier density of $5 \times 10^{12} / \mathrm{cm}^{2}$. Differential transmission signal of quartz substrate is also plotted. (d) Differential transmission signals of CVD grown monolayer graphene measured with grating imaging technique at three excited carrier densities. The solid lines are the fitted curves to the experimental data based on the model described in the text. 


\section{Modeling}

In order to extract the ambipolar diffusion coefficient $D$ from the transient transmission signals, we modified the dynamic differential optical conductivity model [23] by taking the diffusion effect into account. In the dynamic optical conductivity model, the differential transmission signal $\Delta \mathrm{T} / \mathrm{T}_{0}$ for graphene sandwiched by two dielectric medias is proportional to the negative value of the differential real part of optical conductivity $\Delta \sigma=\left(\sigma-\sigma_{0}\right)[21]$ :

$$
\frac{\Delta \mathrm{T}}{\mathrm{T}} \propto-\left(\sigma-\sigma_{0}\right)
$$

The real part of the optical conductivity can be written as $[15,30]$ :

$$
\begin{gathered}
\frac{\sigma}{\sigma_{Q}}=\frac{4 k_{B} T_{e}}{\pi \hbar}\left[\ln \left(1+e^{E_{F}^{h} / k_{B} T_{e}}\right)+\ln \left(1+e^{E_{F}^{e} / k_{B} T_{e}}\right)\right] \frac{1 / \tau_{m}}{\omega^{2}+1 / \tau_{m}^{2}} \\
+\frac{1}{2}\left[\tanh \left(\frac{\hbar \omega-2 E_{F}^{h}}{4 k_{B} T_{e}}\right)+\tanh \left(\frac{\hbar \omega-2 E_{F}^{e}}{4 k_{B} T_{e}}\right)\right]
\end{gathered}
$$

where $\sigma_{Q}$ is the universal quantum conductivity (different from the unexcited one $\sigma_{0}$ ), $T_{e}$ is the electron temperature, $E_{F}^{e}\left(E_{F}^{h}\right)$ is the electron (hole) Fermi energy, and $\tau_{m}$ is the momentum scattering time. It can be seen from Equation (1) and Equation (2) that transient transmission signal is explicitly related to Fermi energy and electron temperature, both of which are associated with carrier density. Therefore, we first calculate transient carrier density excited by the pump pulse by solving the carrier diffusion-recombination equation:

$$
\partial N_{e(h)}(r, t) / \partial t=D \nabla^{2} N_{e(h)}(r, t)-N_{e(h)}(r, t) / \tau_{r}
$$


When there is no grating, only the second term on the right hand matters and the solution of Equation (3) is just an exponential decay with a carrier lifetime $\tau_{r}$; When there is grating, carrier diffusion (the first term) takes effect, resulting in a faster decrease of carrier density in the illuminated area. With grating, considering the initial condition as the excitation with a Gaussian beams modulated by a square wave, Equation (2) can be solved numerically to obtain the carrier density evolution in space and time $N_{e(h)}(r, t)$.

Without grating, electron temperature $T_{e}$ simply follows a decaying bi-exponential function due to energy transfer from hot carriers to optical phonons and acoustic phonons (lattice) $[19,22,31]: T_{e}(t)=T_{0}+\left(T_{e \max }-T_{1}\right) \exp \left(-t / \tau_{o p}\right)+\left(T_{1}-T_{0}\right) \exp \left(-t / \tau_{a p}\right)$, where $T_{0}$ is initial lattice temperature $(300 \mathrm{~K}), T_{e \max }$ denotes the maximum electron temperature when the excited carriers just finish thermalization, $T_{1}$ denotes the equilibrium temperature between the electron system and the optical phonon system, and $\tau_{o p}$ and $\tau_{a p}$ are the times for energy transfer from hot electrons to optical phonons and acoustic phonons (lattice), respectively. $T_{e \max }$ and $T_{1}$ can be calculated from the energy conservations within the electron system and within the coupled electron-optical phonon system, respectively $[15,30,32]$; With grating, the electron temperature will also decay faster due to electron diffusion (energy dissipation) from the illuminated area to the dark area. Considering that the electron-optical phonon scattering time $\tau_{o p}$ in graphene is about tens of fs $[16,19,33]$, within such a short time, electron diffusion has little effect on $T_{e}$. Therefore, even with grating, the maximum electron temperature $T_{e \max }$, the electron-optical phonon equilibrium temperature $T_{1}$, and the cooling 
time to optical phonons $\tau_{o p}$ are expected to be the same as the no-grating case. The only parameter affected substantially by the diffusion effect is the cooling time to acoustic phonons $\tau_{a p}$. So for the case of grating, the dynamic of $T_{e}$ is: $T_{e}(t)=T_{0}+\left(T_{\text {emax }}-T_{1}\right) \exp \left(-t / \tau_{o p}\right)+\left(T_{1}-T_{0}\right) \exp \left(-t / \tau_{\text {diff }}\right)$, with $\tau_{\text {diff }}$ being the modified cooling time due to diffusion.

Since electrons in graphene obey Fermi distribution, the time-dependent Fermi energy $E_{F}^{e}(t)\left(E_{F}^{h}(t)\right)$ is determined from $N_{e(h)}$ and $T_{e}$ by Fermi integral relation in momentum space: $N_{e(h)}(t)=4 \iint d k_{x} d k_{y}(1 / 2 \pi)^{2} /\left\{1+\exp \left[\left(\hbar v|k|-E_{F}^{e(h)}(t)\right) / k_{b} T_{e}(t)\right]\right\}$, where $k$ is electron(hole) wave vector, and $v$ is the Fermi velocity of electrons in graphene [30]. Thus, by substituting $E_{F}^{e}(t)\left(E_{F}^{h}(t)\right)$ and $T_{e}(t)$ into Equation (2), and using the unchanged parameters such as $T_{e \max }, \tau_{o p}$, and $T_{1}$ obtained from the no grating case, our modified differential optical conductivity model can be applied to fit the transient transmission signals $\Delta \mathrm{T} / \mathrm{T}_{0}$, with $D$ and $\tau_{\text {diff }}$ as two fitting parameters.

\section{Results and Discussion}

\subsection{Fitted results}

Fitted curves of transient transmission signals with Equation (2) are presented as solid curves in Fig. 2. It can be seen that for both cases with and without grating cases, experimental data can be well fitted with our model. In the CVD grown 12-layer sample, the fitted values for the no grating case agree well with values reported in lit- 
erature: $\tau_{r}=0.68(0.5[33], 1.3[15], \sim 0.5[16]) \mathrm{ps}, \tau_{m}=3.3(2[15], 5[18], 4[34]) \mathrm{fs}$, $\tau_{o p}=79.6(<100[33], 80-100[16], 70-120[19]) \mathrm{fs}$, and $\tau_{a p}=1.13(1[33], 0.72-0.86[16], 0.4-1.7[19]) p s$. With gratings, our fittings yields $D=2103 \pm 271 \mathrm{~cm}^{2} / \mathrm{s}, \tau_{\text {diff }}=870 \pm 69 \mathrm{fs}$ for $1.5 \mu \mathrm{m}$ grating slit and $D=1901 \pm 245 \mathrm{~cm}^{2} / \mathrm{s}$ $\tau_{\text {diff }}=778 \pm 43 \mathrm{fs}$ for $2.0 \mu \mathrm{m}$ grating slit. For both grating slits, $\tau_{\text {diff }}$ is indeed smaller than $\tau_{a p}$ obtained for the case without grating, confirming the physical picture that electron diffusion also carries energy out of the illuminated area and makes the energy dissipation faster. Considering the uncertainties from both experiments and the fitting model, two fitted coefficients $D$ with two slits are slightly different, but still in the same range, indicating self-consistence of our experimental technique and numerical modeling. The same fitting procedure is performed for the exfoliated 18-layer graphene and the bilayer CVD grown sample, as shown in Fig. 2(b) and Fig. 2(c), respectively. The fitting yields: $D=3489 \pm 397 \mathrm{~cm}^{2} / \mathrm{s}$ for $1.5 \mu \mathrm{m}$ grating slit and $D=3609 \pm 185 \mathrm{~cm}^{2} / \mathrm{s}$ for $2.0 \mu \mathrm{m}$ grating slit, for the 18-layer graphene; and $D=4451 \pm 423 \mathrm{~cm}^{2} / \mathrm{s}$ for $1.5 \mu \mathrm{m}$ grating slit and $D=4067 \pm 386 \mathrm{~cm}^{2} / \mathrm{s}$ for $2.0 \mu \mathrm{m}$ grating slit for the bilayer graphene. For the CVD grown monolayer sample, we measured $D$ at various excited carrier densities from $3.75 \times 10^{12} \sim 21.2 \times 10^{12} / \mathrm{cm}^{2}$, three of which are shown in Fig. 2(d).

The fitted values of $D$ in the CVD grown monolayer sample are plotted in Fig. 3 as a function of carrier density. Within our measured range of laser fluences, $D$ s do not show obvious dependence on carrier density. In fact, there are three major carrier scattering mechanisms in graphene supported with substrate: scattering with acoustic phonons in graphene, Coulomb scattering with ionized impurities, and scattering with 
phonons in substrate. When ionized impurity scattering dominates, carrier mobility and the diffusion coefficient in monolayer graphene are almost constant with carrier density $[4,35]$. As shown in Fig. 3, the diffusion length of our CVD grown monolayer graphene is estimated with the formula $L=\sqrt{D \tau_{r}}$. Since $\tau_{r}$ only decreases slightly with carrier density (Fig. S7), the derived diffusion length is almost constant with carrier density, with a value about $1.4 \mu \mathrm{m}$. Although graphene has a remarkable diffusion coefficient as compared with traditional semiconductors with non-zero bandgap such as $\mathrm{Si}\left(\leq 36 \mathrm{~cm}^{2} / \mathrm{s}\right.$ for electrons [36]) and $\mathrm{GaAs}\left(\leq 200 \mathrm{~cm}^{2} / \mathrm{s}\right.$ for electrons [37]), the carrier lifetime in graphene ( 1ps) is several orders of magnitude shorter, which adversely limits the diffusion length in graphene to be in the order of $\mu \mathrm{m}$, much shorter than those in the traditional semiconductors ( $\mathrm{Si}: 100-300 \mu \mathrm{m}, \mathrm{GaAs}$ : tens of $\mu \mathrm{m}$ ) [38]. The diffusion length obtained here unveils the spatial scale of carrier diffusion in the CVD grown graphene, providing a useful reference for designing future graphene-based opto-electronic devices where ambipolar carrier diffusion would take place. 


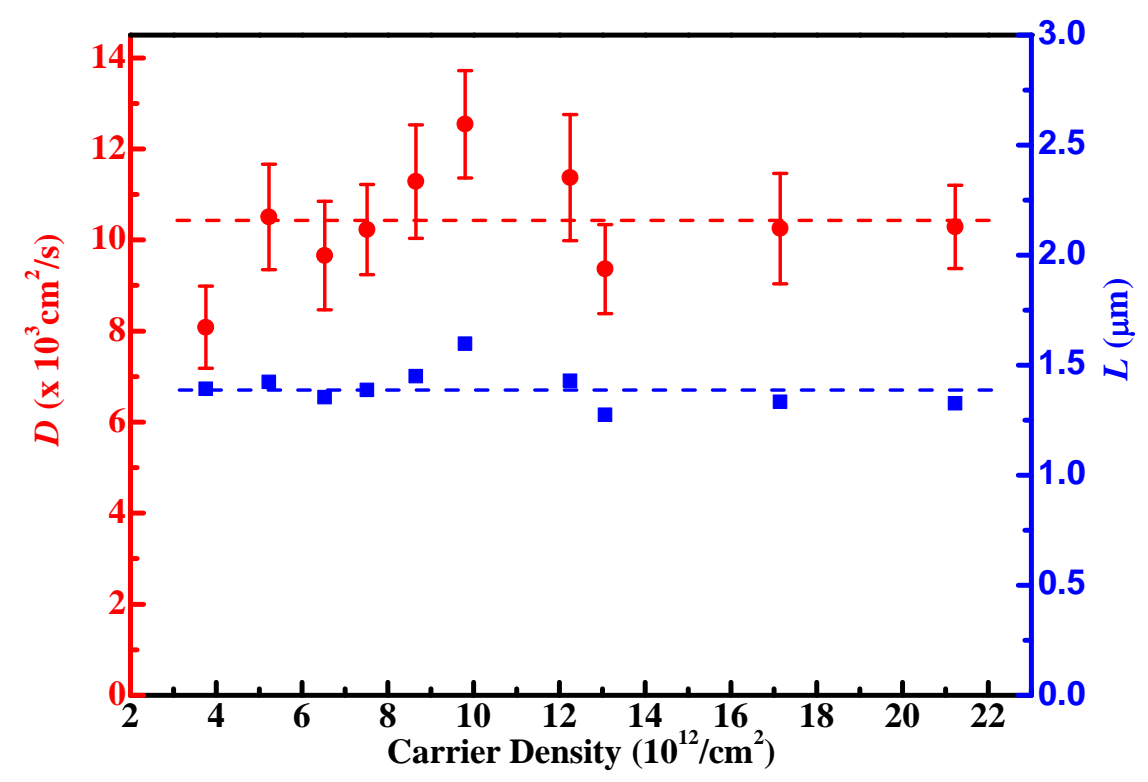

Fig 3. Diffusion coefficient $D$ in CVD grown monolayer graphene at various excited carrier densities. The corresponding diffusion length is estimated from the formula $L=\sqrt{D \tau_{r}}$, with diffusion coefficient $D$ and the carrier lifetime $\tau_{r}$ fitted from experimental data.

\subsection{Discussion}

In literature very few direct measurements of the carrier diffusion coefficient of graphene have been reported. Table 1summarizes the measured $D$ s in graphene prepared with different synthesis methods and on various substrates. In Ref. [24], $D$ were measured with spatial scanning microspectroscopy, with both pump and probe lasers at $800 \mathrm{~nm}$ wavelength. The first observation is that the $D$ in monolayer samples is several times larger than those in fewlayer and multilayer samples, despite different synthesis methods, substrates and measurement techniques. According to microscopic statistic theory, the carrier diffusion coefficient can be calculated as: $D=\left\langle k^{2} \tau_{m} / 2 m^{* 2}\right\rangle$ 
, where $k$ is the electron momentum, $\tau_{m}$ is the momentum scattering time, $m^{*}$ is electron effective mass, and the bracket " $<>$ " means averaging over $k$ [39].For all the measured $D$ s presented in Table 1, both pump and probe pulses have wavelengths at 800nm, hence similar distributions of electron momentum are expected. For CVD grown samples, our fitting results show that $\tau_{m}$ of the multilayer $(\sim 3 \mathrm{fs})$ and bilayer $(\sim 2 \mathrm{fs})$ are larger than that of the monolayer ( 1fs) (Fig. S8). $\tau_{m}$ reflects the effect of scattering on carriers. In the multilayer sample, the inner layers are protected from the charged impurity scattering at $\mathrm{SiO}_{2}$ interface [4], which results in longer scattering time. Since $\tau_{m}$ tends to produce a larger $D$ in multilayer sample, it cannot explain the trend observed in Table1. Another parameter that can play an important role is the effective mass $m^{*}$ [39]. In monolayer graphene, the energy band has a unique Dirac cone structure, and consequently, the electrons near the cone are nearly massless, $m^{*} \sim 0$. In the bilayer or multilayer graphene, the energy structure is affected and becomes a wider paraboloid around the $\mathrm{K}\left(\mathrm{K}^{\prime}\right)$ point due to interlayer coupling [40-42], which results in a finite effective mass around $K\left(K^{\prime}\right)$ point [43]. Larger average effective mass in bilayer and multilayer graphene even rivals the counter effect from $\tau_{m}$ and brings about smaller $D$ compared to the monolayer graphene. Actually, this effective mass variation mechanism has been proposed [44-46] and can be used to explain the layer number dependence of Hall and Field-Effect mobility $\mu$ measured in graphene [44-49]. 
Table 1.

Carrier diffusion coefficients of graphene samples prepared with different synthesis methods and on various substrates

\begin{tabular}{ccccccc}
\hline Synthesis Method & CVD & Epitaxial & CVD & Exfoliated & CVD & $\begin{array}{c}\text { Thermally } \\
\text { reduced }\end{array}$ \\
\hline Layer No. & 1 & 1 & 2 & 18 & 12 & 50 \\
\hline Substrate & quartz & $\mathrm{SiC}$ & quartz & quartz & quartz & quartz \\
\hline $\boldsymbol{D}\left(\mathbf{c m}^{2} / \mathbf{s}\right)$ & $\begin{array}{c}10000 \\
\text { This work }\end{array}$ & $\begin{array}{c}10000 \\
\text { Ref. 24 }\end{array}$ & $\begin{array}{c}4250 \\
\text { This work }\end{array}$ & $\begin{array}{c}3500 \\
\text { This work }\end{array}$ & $\begin{array}{c}2000 \\
\text { This work }\end{array}$ & $\begin{array}{c}1300 \\
\text { Ref. } 24\end{array}$ \\
\hline
\end{tabular}

The second observation from Table 1 is that $D$ measured in the exfoliated multilayer sample is much larger than that in the CVD grown multilayer sample, on the same substrates and with similar number of layers. Since these two samples have similar layer numbers and substrates, it is reasonable to expect that scatterings with intrinsic acoustic phonons and with substrate phonons are similar in both of them. The observed difference in $D$ s very likely comes from the difference in impurity scattering. Multilayer graphene exfoliated from high-quality natural graphite usually has much less impurities, while CVD grown graphene usually has many growth-induced defects and impurities, such as the disrupted $\mathrm{sp}^{2}$ bond of point defects and grain boundaries $[50,51]$, and the chemical residue [52]. Therefore, it is reasonable to expect a higher defects concentration in CVD grown multilayer graphene and thus a smaller $D$. The 50-layer thermally reduced graphene has the lowest $D$ among all samples, which could result from a combined effect of layer number and high impurity concentration [53].

Using the Einstein relation, $D=k_{b} \bar{T}_{e} \mu / q$, the hot carrier mobility $\mu$ of our three 
CVD samples are estimated with measured $D$, shown as the red dots in Fig. 4. $\bar{T}_{e}$ is the average electron temperature calculated from our model, $750 \mathrm{~K}, 650 \mathrm{~K}$, and $650 \mathrm{~K}$ for monolayer, bilayer and multilayer graphene, respectively (Fig. S6). For comparison, reported values of Hall mobility and Field-Effect mobility in exfoliated graphene on $\mathrm{SiO}_{2} / \mathrm{Si}$ substrates are also plotted in Fig. 4, against layer number. All the measured mobilities show strong dependence on layer number. Interestingly, substantial decrease is only observed between monolayer and bilayer graphene, while the difference among bilayer and other multilayer graphene is not as obvious. This observation supports that the exotic electronic properties of monolayer graphene are mainly due to its unique 2D band structure, which could not be achieved in multilayer graphene. Another striking feature shown in Fig. 4 is that, hot carrier mobility deduced from optical measurement $\left(\sim 36,000 \mathrm{~cm}^{2} / \mathrm{Vs}\right.$ for multilayer graphene, $\sim 72,000 \mathrm{~cm}^{2} / \mathrm{Vs}$ for bilayer and $\sim 120,000 \mathrm{~cm}^{2} / \mathrm{Vs}$ for monolayer graphene) are an order of magnitude larger than Hall Mobility and Field-Effect mobility. The distinct difference between the optically measured and the electrically measured mobilities has also been reported in epitaxial graphene on $\mathrm{SiC}$ in Ref. [24] and CVD grown graphene on $\mathrm{SiO}_{2}$ in Ref. [25]. Our measured $D$ s in graphene supports that ionized impurity scattering dominates carrier scattering process in graphene, which has also been demonstrated in the resistivity measurement of graphene Field-Effect transistors [1]. Ionized impurities can gradually deviate the charged carrier trajectory by Coulomb potential and the scattering cross section $\sigma$ is inversely proportional to the square of charged carrier energy $E: \sigma \sim 1 / E^{2}$ [54]. In un-doped non-degenerate semiconductors, mobility due to ionized impurity 
scattering has a temperature dependence of $\sim \bar{T}_{e}^{3 / 2}$ [5]. Since photoexcited carriers have much higher energy than electrically excited ones, the scattering cross section of photoexcited carriers due to ionized impurities is expected to be much smaller, which brings about a larger mobility measured in this work than Hall mobility and Field-Effect mobility.

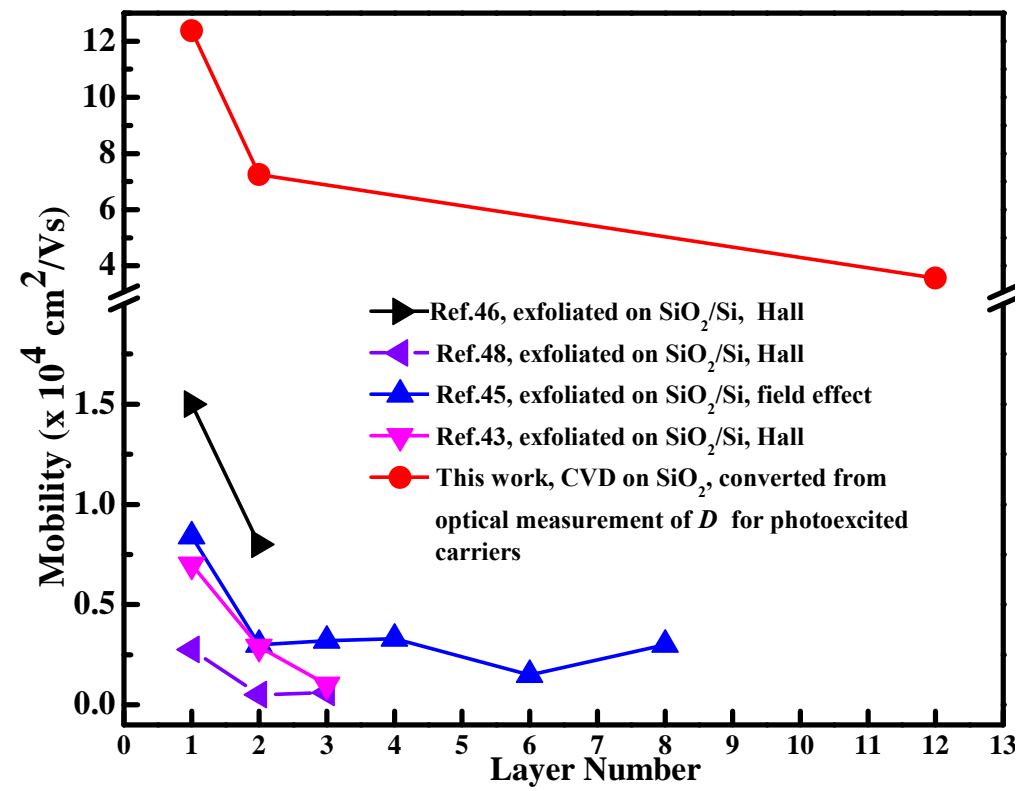

Fig. 4.Carrier mobility as a function of graphene layer number. The triangles are reported Hall mobility and Field-Effect mobility. The red dotsarethe hot carrier mobility deduced from our experimental data using the Einstein relation $D / \mu=k_{b} \bar{T}_{e} / q$.

Our results have several important implications: a) Layer-number dependence, mainly the difference between quadratic energy structure in multilayer graphene and the linear energy structure in monolayer graphene, has significant influence on carrier transport properties. To realize high-performance graphene devices, synthesis of true mono-layer graphene is indispensable. b) Charged impurity scattering dominates in both monolayer and multilayer samples. c) Transport properties of photoexcited hot carriers are remarkably different from that of electrically induced room-temperature 
carriers. When evaluating performance of graphene-based opto-eletronics, it is necessary to use transport properties of photoexcited carriers, instead of room-temperature ones.

\section{CONCLUSION}

An ultrafast grating imaging technique has been utilized to measure transport properties $(\boldsymbol{\mu}, \boldsymbol{L}, \boldsymbol{D})$ of photoexcited carriers in graphene. All the transport properties do not show obvious dependence on carrier density, which supports the fact that ionized impurity scattering dominates the carrier scattering process in graphene. $\boldsymbol{D}$ and $\boldsymbol{\mu}$ show drastic decrease from monolayer to multilayer samples, which was attributed to the unique Dirac Cone energy structure in monolayer graphene. The measured mobilities of photoexcited carriers are several times larger than that of Hall and Field-Effect mobilities, due to the much high carrier energy of optically excited hot carriers. Our results indicate the importance of obtaining monolayer graphene to realize high-performance graphene devices, as well as the necessity to use transport properties of photoexcited carriers for predicting the performance of graphene-based opto-electronics. 


\section{ACKNOWLEDGMENT}

The authors are grateful for the support from National Science Foundation (CARER, Grant No. CBET-1351881, and NASCENT, Grant No. EEC-1160494).T. S. Lai also acknowledges the support from National Basic Research Program of China under Grant No.2013CB922403, National Natural Science Foundation of China under Grant Nos. 11274399 and 61475195, Natural Science Foundation of Guangdong Province under Grant No.2014A030311029

\section{Reference}

[1] K.S. Novoselov, A.K. Geim, S.V. Morozov, D. Jiang, M.I. Katsnelson, I.V. Grigorieva, S.V. Dubonos, A.A. Firsov, Two-dimensional gas of massless Dirac fermions in graphene, Nature 438(7065) (2005) 197-200.

[2] K.S. Novoselov, A.K. Geim, S.V. Morozov, D. Jiang, Y. Zhang, S.V. Dubonos, I.V. Grigorieva, A.A. Firsov, Electric field effect in atomically thin carbon films, Science 306(5696) (2004) 666-669.

[3] Y.B. Zhang, Y.W. Tan, H.L. Stormer, P. Kim, Experimental observation of the quantum Hall effect and Berry's phase in graphene, Nature 438(7065) (2005) 201-204.

[4] J.H. Chen, C. Jang, S.D. Xiao, M. Ishigami, M.S. Fuhrer, Intrinsic and extrinsic performance limits of graphene devices on SiO2, Nat Nanotechnol 3(4) (2008) 206-209.

[5] P.Y. Yu, M. Cardona, Fundamentals of Semiconductors: Physics and Materials Properties, Third, Revised and Enlarged Edition, Springer Press (2003) 220-235.

[6] C.M. Corbett, C. McClellan, A. Rai, S.S. Sonde, E. Tutuc, S.K. Banerjee, Field Effect Transistors with Current Saturation and Voltage Gain in Ultrathin ReS2, Acs Nano 9(1) (2015) 363-370.

[7] Z. Liu, L.L. Ma, G. Shi, W. Zhou, Y.J. Gong, S.D. Lei, X.B. Yang, J.N. Zhang, J.J. Yu, K.P. Hackenberg, A. Babakhani, J.C. Idrobo, R. Vajtai, J. Lou, P.M. Ajayan, In-plane heterostructures of graphene and hexagonal boron nitride with controlled domain sizes, Nat Nanotechnol 8(2) (2013) 119-124. [8] M.P. Levendorf, C.J. Kim, L. Brown, P.Y. Huang, R.W. Havener, D.A. Muller, J. Park, Graphene and boron nitride lateral heterostructures for atomically thin circuitry, Nature 488(7413) (2012) 627-632. 
[9] G. Fiori, A. Betti, S. Bruzzone, G. lannaccone, Lateral Graphene-hBCN Heterostructures as a Platform for Fully Two-Dimensional Transistors, Acs Nano 6(3) (2012) 2642-2648.

[10] J.Q. He, N. Kumar, M.Z. Bellus, H.Y. Chiu, D.W. He, Y.S. Wang, H. Zhao, Electron transfer and coupling in graphene-tungsten disulfide van der Waals heterostructures, Nat Commun 5 (2014) 5622. [11] A. Boudjani, G. Bassou, T. Benbakhti, M. Beghdad, B. Belmekki, Direct Measurement of Minority-Carrier Diffusion Length in Planar Devices, Solid State Electron 38(2) (1995) 471-475.

[12] J. Navas, E. Guillen, R. Alcantara, C. Fernandez-Lorenzo, J. Martin-Calleja, G. Oskam, J. Idigoras, T. Berger, J.A. Anta, Direct Estimation of the Electron Diffusion Length in Dye-Sensitized Solar Cells, J Phys Chem Lett 2 (2011) 1045-1050.

[13] J.W. Orton, P. Blood, The Electrical Characterization of Semiconductors: Measurement of Minority Carrier Properties, Academic Press, London (1990) 217-278.

[14] F. Bonaccorso, Z. Sun, T. Hasan, A.C. Ferrari, Graphene photonics and optoelectronics, Nat Photonics 4(9) (2010) 611-622.

[15] H. Choi, F. Borondics, D.A. Siegel, S.Y. Zhou, M.C. Martin, A. Lanzara, R.A. Kaindl, Broadband electromagnetic response and ultrafast dynamics of few-layer epitaxial graphene, Appl Phys Lett 94 (2009) 172102.

[16] I. Gierz, J.C. Petersen, M. Mitrano, C. Cacho, I.C.E. Turcu, E. Springate, A. Stohr, A. Kohler, U. Starke, A. Cavalleri, Snapshots of non-equilibrium Dirac carrier distributions in graphene, Nat Mater 12(12) (2013) 1119-1124.

[17] T. Limmer, J. Feldmann, E. Da Como, Carrier Lifetime in Exfoliated Few-Layer Graphene Determined from Intersubband Optical Transitions, Phys Rev Lett 110 (2013) 217406.

[18] J.H. Strait, H.N. Wang, S. Shivaraman, V. Shields, M. Spencer, F. Rana, Very Slow Cooling Dynamics of Photoexcited Carriers in Graphene Observed by Optical-Pump Terahertz-Probe Spectroscopy, Nano Lett 11(11) (2011) 4902-4906.

[19] J.M. Dawlaty, S. Shivaraman, M. Chandrashekhar, F. Rana, M.G. Spencer, Measurement of ultrafast carrier dynamics in epitaxial graphene, Appl Phys Lett 92 (2008) 042116.

[20] P. Plochocka, P. Kossacki, A. Golnik, T. Kazimierczuk, C. Berger, W.A. de Heer, M. Potemski, Slowing hot-carrier relaxation in graphene using a magnetic field, Phys Rev B 80 (2009) 245415.

[21] L.M. Malard, K.F. Mak, A.H.C. Neto, N.M.R. Peres, T.F. Heinz, Observation of intra- and inter-band transitions in the transient optical response of graphene, New J Phys 15 (2013) 015009.

[22] L.B. Huang, G.V. Hartland, L.Q. Chu, Luxmi, R.M. Feenstra, C.X. Lian, K. Tahy, H.L. Xing, Ultrafast Transient Absorption Microscopy Studies of Carrier Dynamics in Epitaxial Graphene, Nano Lett 10(4) (2010) 1308-1313.

[23] K. Chen, H.H. Li, L.P. Ma, W.C. Ren, T.F. Chung, H.M. Cheng, Y.P. Chen, T.S. Lai, Diversity of ultrafast hot-carrier-induced dynamics and striking sub-femtosecond hot-carrier scattering times in graphene, Carbon 72 (2014) 402-409.

[24] B.A. Ruzicka, S. Wang, L.K. Werake, B. Weintrub, K.P. Loh, H. Zhao, Hot carrier diffusion in graphene, Phys Rev B 82 (2010) 195414.

[25] B.A. Ruzicka, S. Wang, J.W. Liu, K.P. Loh, J.Z. Wu, H. Zhao, Spatially resolved pump-probe study of single-layer graphene produced by chemical vapor deposition [Invited], Opt Mater Express 2(6) (2012) 708-716.

[26] A.R. Cameron, P. Riblet, A. Miller, Spin gratings and the measurement of electron drift mobility in multiple quantum well semiconductors, Phys Rev Lett 76 (1996) 4793-4796.

[27] H. Hillmer, A. Forchel, S. Hansmann, M. Morohashi, E. Lopez, H.P. Meier, K. Ploog, Optical 
Investigations on the Mobility of Two-Dimensional Excitons in Gaas/Ga1-Xalxas Quantum Wells, Phys Rev B 39 (1989) 10901-10912.

[28] K. Chen, W.F. Wang, J.M. Chen, J.H. Wen, T.S. Lai, A transmission-grating-modulated pump-probe absorption spectroscopy and demonstration of diffusion dynamics of photoexcited carriers in bulk intrinsic GaAs film, Opt Express 20(4) (2012) 3580-3585.

[29] Y. Huang, E. Sutter, N.N. Shi, J. Zheng, T. Yang, D. Englund, H.J. Gao, P. Sutter, Reliable Exfoliation of Large-Area High-Quality Flakes of Graphene and Other Two-Dimensional Materials, Acs Nano 9(11) (2015) 10612-20.

[30] K.M. Dani, J. Lee, R. Sharma, A.D. Mohite, C.M. Galande, P.M. Ajayan, A.M. Dattelbaum, H. Htoon, A.J. Taylor, R.P. Prasankumar, Intraband conductivity response in graphene observed using ultrafast infrared-pump visible-probe spectroscopy, Phys Rev B 86 (2012) 125403.

[31] J.Z. Shang, T. Yu, J.Y. Lin, G.G. Gurzadyan, Ultrafast Electron-Optical Phonon Scattering and Quasiparticle Lifetime in CVD-Grown Graphene, Acs Nano 5(4) (2011) 3278-3283.

[32] T. Li, L. Luo, M. Hupalo, J. Zhang, M.C. Tringides, J. Schmalian, J. Wang, Femtosecond Population Inversion and Stimulated Emission of Dense Dirac Fermions in Graphene, Phys Rev Lett 108 (2012) 167401.

[33] C.H. Lui, K.F. Mak, J. Shan, T.F. Heinz, Ultrafast Photoluminescence from Graphene, Phys Rev Lett 105 (2010) 127404.

[34] J.M. Dawlaty, S. Shivaraman, J. Strait, P. George, M. Chandrashekhar, F. Rana, M.G. Spencer, D. Veksler, Y.Q. Chen, Measurement of the optical absorption spectra of epitaxial graphene from terahertz to visible, Appl Phys Lett 93 (2008) 131905.

[35] E.H. Hwang, S. Adam, S. Das Sarma, Carrier transport in two-dimensional graphene layers, Phys Rev Lett 98 (2007) 186806.

[36] R. Brunetti, C. Jacoboni, F. Nava, L. Reggiani, G. Bosman, R.J.J. Zijlstra, Diffusion-Coefficient of Electrons in Silicon, J Appl Phys 52 (1981) 6713-6722.

[37] M. Demurcia, D. Gasquet, A. Elamri, J.P. Nougier, J. Vanbremeersch, Diffusion and Noise in Gaas Material and Devices, leee T Electron Dev 38(11) (1991) 2531-2539.

[38] A. Dargys, J. Kundrotas, Handbook on Physical Properties of Ge, Si, GaAs and InP, Vilnius, Science and Encyclopedia Publishers (1994).

[39] M.W. Wu, J.H. Jiang, M.Q. Weng, Spin dynamics in semiconductors, Phys Rep 493(2-4) (2010) 61-236.

[40] H.K. Min, A.H. MacDonald, Chiral decomposition in the electronic structure of graphene multilayers, Phys Rev B 77 (2008) 155416.

[41] B. Partoens, F.M. Peeters, From graphene to graphite: Electronic structure around the K point, Phys Rev B 74 (2006) 075404.

[42] J.B. Oostinga, H.B. Heersche, X.L. Liu, A.F. Morpurgo, L.M.K. Vandersypen, Gate-induced insulating state in bilayer graphene devices, Nat Mater 7(2) (2008) 151-157.

[43] H. Raza, E.C. Kan, Armchair graphene nanoribbons: Electronic structure and electric-field modulation, Phys Rev B 77 (2008) 245434.

[44] K. Nagashio, T. Nishimura, K. Kita, A. Toriumi, Mobility Variations in Mono- and Multi-Layer Graphene Films, Appl Phys Express 2 (2009) 025003.

[45] K. Nagashio, T. Nishimura, K. Kita, A. Toriumi, Systematic Investigation of the Intrinsic Channel Properties and Contact Resistance of Monolayer and Multilayer Graphene Field-Effect Transistor, Jpn J Appl Phys 49 (2010) 051304. 
[46] M.F. Craciun, S. Russo, M. Yamamoto, J.B. Oostinga, A.F. Morpurgo, S. Tarucha, Trilayer graphene is a semimetal with a gate-tunable band overlap, Nat Nanotechnol 4(6) (2009) 383-388.

[47] L. Wang, X.S. Chen, A.Q. Yu, Y. Zhang, J.Y. Ding, W. Lu, Highly Sensitive and Wide-Band Tunable Terahertz Response of Plasma Waves Based on Graphene Field Effect Transistors, Sci Rep-Uk 4 (2014) 5470.

[48] W.J. Zhu, V. Perebeinos, M. Freitag, P. Avouris, Carrier scattering, mobilities, and electrostatic potential in monolayer, bilayer, and trilayer graphene, Phys Rev B 80 (2009) 235402.

[49] S.V. Morozov, K.S. Novoselov, M.I. Katsnelson, F. Schedin, D.C. Elias, J.A. Jaszczak, A.K. Geim, Giant intrinsic carrier mobilities in graphene and its bilayer, Phys Rev Lett 100 (2008) 016602.

[50] H.S. Song, S.L. Li, H. Miyazaki, S. Sato, K. Hayashi, A. Yamada, N. Yokoyama, K. Tsukagoshi, Origin of the relatively low transport mobility of graphene grown through chemical vapor deposition, Sci Rep-Uk 2 (2012) 337.

[51] F. Banhart, J. Kotakoski, A.V. Krasheninnikov, Structural Defects in Graphene, Acs Nano 5(1) (2011) 26-41.

[52] A. Pirkle, J. Chan, A. Venugopal, D. Hinojos, C.W. Magnuson, S. McDonnell, L. Colombo, E.M. Vogel, R.S. Ruoff, R.M. Wallace, The effect of chemical residues on the physical and electrical properties of chemical vapor deposited graphene transferred to SiO2, Appl Phys Lett 99 (2011) 122108.

[53] H.J. Kim, D. Kim, S. Jung, S.N. Yi, Y.J. Yun, S.K. Chang, D.H. Ha, Charge Transport in Thick Reduced Graphene Oxide Film, J Phys Chem C 119 (2015) 28685-28690.

[54] E. Conwell, V.F. Weisskopf, Theory of Impurity Scattering in Semiconductors, Phys Rev 77 (1950) 388-390. 